\title{
SERIES DE FICCIÓN COMO MEDIO DE COEDUCACIÓN PARA ADOLES- CENTES. ESTUdiO DE CASO: LAS DEL HOCKEY
}

\section{Fiction Series as a Means for the Co-Education of Teenagers. Report of a case: The Girls Hockey}

\section{Dr. Javier GIL-QUINTANA}

Universidad Nacional de Educación a Distancia, España

E-mail: jgilquintana@edu.uned.es

(iD) http://orcid.org/0000-0003-0326-2535

\author{
Gr. Simón GIL-TÉVAR \\ Universidad Nacional de Educación a Distancia, España \\ E-mail: simon.comunica@gmail.com \\ (iD) http://orcid.org/0000-0003-3313-0218
}

Fecha de recepción del artículo: 10/09/2020

Fecha de aceptación definitiva: 15/10/2020

\begin{abstract}
RESUMEN
La serie "Las del Hockey» (The Girls hockey/ Les de l'hoquei) es una creación nacida de un Trabajo final del grado de Comunicación Audiovisual de la Universidad Pompeu Fabra (Barcelona), creada por Ona Anglada, Laura Azemar, Natalia Boadas y Marta Vivet. La serie ha sido producida por Brutal Media y emitida también por TV3. A través del método de estudio de caso analizamos la serie "Las del Hockey" desde una metodológica mixta haciendo, uso de instrumentos como las entrevistas semiestructuradas a observadoras privilegiadas, estudio de nueve perfiles de la red social Instagram, análisis de contenido de trece capítulos de la serie emitidos en la primera temporada y de la audiencia, comparando la primera y segunda temporada. Los resultados de nuestra investigación ponen de manifiesto que las series de ficción han sido testigo de la evolución social hacia la igualdad de género y en la adquisición de derechos de la mujer en la sociedad. A pesar de ello, en numerosas producciones se proyectan roles y estereotipos de género basados en la discriminación. Es imprescindible incentivar en todos los espacios educativos formales y no formales una coeducación mediática que permita el desarrollo de un pensamiento crítico, de una actitud crítica.
\end{abstract}

Palabras clave: Coeducación; igualdad de género; adolescentes; series de ficción; educomunicación; media literacy.

\section{ABSTRACT}

The TV series "Las del Hockey" (The Girls hockey / Les de l'hoquei) is a creation born from a final degree project in Audiovisual Communication at the Pompeu Fabra University (Barcelona), created by Ona Anglada, Laura Azemar, Natalia Boadas and Marta Vivet. 
The TV series has been produced by Brutal Media and has been also broadcast in TV3. Through a case study method, we analyze the TV series "Las del Hockey» with a mixed methodology. We use instruments such as semi-structured interviews with privileged observers, a study of nine profiles in the social network Instagram, a content analysis of thirteen chapters the TV series aired in its first season and of the audience, comparing the first and second seasons. The results of our research show that fiction TV series have witnessed the social evolution towards gender equality and the acquisition of women's rights in society. Despite this, numerous productions include gender roles and stereotypes based on discrimination. It is essential to promote media co-education in all formal and non-formal educational spaces to encourage the development of a critical thinking and a critical attitude.

Key words: co-education; gender equality; teenagers; fiction series; education; communication.

\section{Introducción}

En los últimos años hemos asistido a un creciente aumento de series de ficción protagonizadas por adolescentes, muchas de ellas dirigidas a un público más amplio, y otras a este, aún más específico. A pesar de la aparente modernidad que presentan hacia aquellos destinatarios que viven en ilusiones juveniles, consumen medios y generan aprendizajes transmedia (Gil-Quintana y Osuna-Acedo, 2020), estas series continúan reproduciendo desigualdad de género, convirtiéndose, una vez más, en una demanda para los procesos de alfabetización mediática y el planteamiento de la coeducación (Belmonte y Guillamón, 2008). Los medios contribuyen en la creación o extensión de realidades o imaginarios sociales a través de los discursos y la comunicación simbólica. En esta misma línea es importante resaltar la "Teoría del Cultivo», en la cual, se plantea que los medios, y tradicionalmente la propia televisión, favorece la estandarización de roles y comportamientos (Gerbner, Gross, Morgan, y Signorielli, 1986). Las series de ficción permiten una mayor extensión narrativa de los personajes y de las tramas gracias a su acercamiento al público, adicción a su consumo y a su duración proyectada en semanas y temporadas. Son numerosas las horas, en las cuales, el público como espectador establece un vínculo de influencia con los personajes del mundo de la ficción, guardando similitud aquellos que establecemos en nuestro entorno. Esta relación con los personajes de ficción puede influir y moldear la percepción del mundo real en la infancia, adolescencia y juventud (Bermejo, 2012; Osuna-Acedo, Gil-Quintana, y Cantillo-Valero, 2018).

A pesar de los avances en materia de igualdad y la gran promoción que, en la actualidad, está teniendo el movimiento feminista, aún queda mucho por avanzar en el ámbito de la coeducación en los medios. Aún podemos observar, en el universo mediático, rasgos de una representación estereotipada de género basada en la desigualdad (Belmonte y Guillamón, 2008). Los roles femeninos en series de ficción, consumidas por el público adolescente, han evolucionado, pudiéndose apreciar diversos papeles de mujeres con mayor diversidad, siendo más independientes, con estudios y una vida de éxito laboral. Pese a las mejoras, estos papeles ubicados continúan siendo situados en el ámbito privado, permaneciendo tanto emocionalmente como sentimentalmente a la relación con el hombre (Chicharro, 2013).

Uno de los detonantes de esa proliferación de series han sido las plataformas de contenidos digitales (video of demand) como Netflix, HBO o Amazon Prime. Son 
numerosas las producciones protagonizadas por adolescentes y de diversas temáticas como "Stranger Things" (Netflix, 2016-2020), "The OA» (Netflix, 2016-2019) o "Las escalofriantes aventuras de Sabrina» (Netflix, 2019-2020), siendo éxitos que han agrupado a diferentes públicos en frente de las múltiples pantallas. Las series de ficción permiten visibilizar temáticas más complejas y significativas a través de su desarrollo, pudiendo contextualizar o denunciar situaciones que pueden ser temas de debate en la sociedad. Algunas series denuncian situaciones de violencia contra la mujer como el "Cuento de la Criada» (HBO, 2017-2020) o "Big Little Lies» (HBO, 2017-2019); en la misma línea de denuncia, y protagonizadas por adolescentes, nos encontramos con la miniserie "Creedme" (Netflix, 2019); también relacionado con otros temas como el acoso escolar o el suicidio se han creado series como "13 Reasons Why" (Netflix, 2017-2020), o en relación con el racismo se ha presentado "Así nos ven»(Netflix, 2019). Como ocurre con todo el ámbito mercantil, todas estas producciones tratan temas demandados en la sociedad (Bonavitta y De Garay, 2019); por ello, plataformas como Netflix han acogido a series que de éxito en televisión (TV3), que versan sobre temas de denuncia social como "Merlí» (Tv3, 2015-2018), o la serie objeto de nuestro estudio como es "Las del Hockey» (Brutal Media y Tv3, 2018-2020).

Al igual que los medios cooperan en procesos de manipulación y creación de identidades sociales creadas también pueden convertirse en un gran instrumento educativo. Existe una tendencia creciente para desarrollar estrategias de alfabetización mediáticas consideradas como "edu-entretenimiento", es decir, introducir contenidos educativos en la emisión de series u otros formatos narrativos, favoreciendo actitudes prosociales y fomentando el cambio de determinados comportamientos alejados del ámbito de los valores y los derechos humanos (Igartua y Vega, 2014; Tufte, 2008). A su vez, se han convertido en un punto de encuentro de socialización, interacción e interrelación, compartiendo experiencias y comentarios desde diversos ámbitos como el familiar y a través de las redes sociales (Gavilán, Martínez-Navarro, y Ayestarán, 2019), incluyendo el desarrollo de una visión transmedia.

En esta investigación presentamos la serie coral de "Las del Hockey» (The Girls bockey/Les de l'hoquei). Se trata de una creación que nació de un «Trabajo final de grado" (TFG) de los estudios de Comunicación Audiovisual de la Universidad Pompeu Fabra (Barcelona), creada por Ona Anglada, Laura Azemar, Natalia Boadas y Marta Vivet. Además de las coautoras se unirían al trabajo del guión Núria Parera y Marta Grau. La serie fue producida por Brutal Media y TV3, y emitida por ésta última. La plataforma Netflix la ha incluido en su catálogo como hiciera con «Merlí»; TV3 renovó para la segunda temporada. Nuestro trabajo se centra en la primera temporada, dada su presencia en abierto en canal autonómico TV3, teniendo en cuenta su inclusión en la plataforma digital de Netflix. La primera temporada consta de 13 capítulos. Los primeros ocho episodios tienen el nombre de una protagonista, teniendo cierta relevancia, con la finalidad de repartir los pesos y mostrar su importancia en la narrativa de la serie. La coralidad de la serie permite una estructura multitrama con diversos personajes singulares en roles de protagonistas (Ruíz y Pérez-Rufí, 2020). La producción está protagonizada por siete adolescentes y su entrenadora, que compiten en el equipo de su pueblo de hockey sobre patines: «Minerva». Las autoras de la serie querían mostrar aquellos aspectos que les hubiera gustado ver cuando eran más jóvenes, reivindicando la adolescencia femenina y los conflictos que les rodean desde una perspectiva de chicas, muchas veces con escasa o mala representación en las series de ficción de adolescentes (Anglada, et al., 2016). Resaltan los referentes de 
la serie en diferentes aspectos; ejemplos como "Orange Is the Black»(2013-2019) o "Transparent»(2014-2017) por su tratamiento a minorías, "Girls»(2012-2017) por una visión femenina de la sociedad, "Friday Night Lights» (2006-2011) por las tramas relacionadas con el deporte y la adolescencia, y otras series adolescentes como "Skins»( 2007-2013), "Misfits»( 2009-2013), "Freaks \& Geeks!»( 1999-2000) o «Banana»( 2015). La serie fue premiada por la Asociación de Mujeres Cineastas y de Medios Audiovisuales para recibir el II Premio TV CIMA a la igualdad. En el reconocimiento se puso en valor la innovación en sus planteamientos, con clara vocación feminista, concebida, escrita y dirigida por mujeres, abordando temáticas relacionadas con la identidad sexual, la inmigración, la adolescencia, pero con una relevante dimensión de la perspectiva de género. Páginas de base de datos especializadas en cine y series como IMDb (Internet Movie Database) le han otorgado una puntación media de 7,1/10; FilmAffinity otorga una puntuación media de 6/10.

Son numerosos los avances en igualdad y en la lucha contra la violencia de género. A pesar de los esfuerzos, según los datos ofrecidos por Ministerio de Igualdad, el año fueron asesinadas 55 mujeres en España, con diferentes edades o condiciones sociales. En base a esta problemática que debe presentarse como una lacra social, diferentes estudios han tratado la coeducación, estereotipos, construcción (Galán, 2006), roles de género de la mujer en la televisión (Belmonte y Guillamón, 2008), en el cine de género deportivo (Ramírez-Macías, Ries, Piedra, y Rodríguez., 2011), en las series de ficción juveniles (García-Muñoz y Fedele, 2011) y en otros géneros de series (Bonavitta y De Garay, 2019; Gavilán, Martínez-Navarro, y Ayestarán, 2019; Raya, Sánchez-Labella, y Durán, 2018). Las aportaciones de investigadoras e investigadores sobre la influencia de las series son la base para vencer la violencia machista desde la coeducación (Perelló, 2019; Subirats, 2019). Nuestro estudio pretende formar parte de esta apuesta por la igualdad y, a través de un estudio de caso, analizar la serie "Las del Hockey" desde una perspectiva metodológica mixta haciendo uso de instrumentos como nueve entrevistas semiestructuradas a observadoras privilegiadas y a una coautora, análisis de nueve perfiles de la red social Instagram, análisis de contenido de doce horas desarrolladas a lo largo de trece capítulos y estudio de la audiencia comparando la primera y segunda temporada. Abrir las puertas a la coeducación audiovisual (Belmonte, 2014) es educar la mirada, poniendo en valor un pensamiento crítico y, por ende, una actitud crítica sobre las representaciones de género discriminatorias basadas en la desigualdad. Una herramienta imprescindible para reflexionar y concienciar al público adolescentes sobre la construcción de identidades y configuraciones de género en una sociedad cada vez más plural. La influencia de medios de los medios y su proyección a través de las series ponen relevancia la necesidad de una alfabetización mediática que proporcione una mirada coeducativa.

\section{Metodología}

El estudio de caso por el que hemos optado en este artículo se trata de un tipo objeto, específico, típico, híbrido, analítico basado en hipótesis y único (Coller, 2005). Este planteamiento de investigación en comunicación y educación (Gil-Quintana, Parejo, y Cantillo-Valero, 2020) parte con el objetivo de dar cuenta del caso basado en la serie "Las del Hockey» con el fin de conocerlo mejor, utilizando el método científico y el conocimiento mediático para interpretarlo. La elección del caso propuesto viene 
determinada por ser una ejemplificación clara y manifiesta sobre los parámetros que debería tener una serie coeducativa como recurso. Optar por una metodología mixta convierte en nuestro estudio en una propuesta que se enriquece de técnicas cualitativas y cuantitativas que ayudan a los investigadores en la recogida y recopilación de los datos, otorgando importancia a la información obtenida de cada una de las funciones. El papel de estos agentes en el estudio trata de posibilitar al lector de este artículo la visualización de las producciones mediáticas desde una visión simplista, colaborar en su interpretación y en la generación de un nuevo conocimiento (Stake, 2010).

Con la finalidad de estudiar las series de ficción como medio coeducativo y, concretamente, el estudio del caso propuesto, hemos procedido a un análisis de contenido desde un enfoque mixto, utilizando la naturaleza cuantitativa de las tablas de estimaciones basadas en la frecuencia de las observaciones, mientras que los aspectos cualitativos de las entrevistas semiestructuradas nos han permitido ahondar más en el propio análisis del mismo, así como la 'minería de textos' extraído de otros estudios nos ha permitido realizar un diagnóstico sobre la coeducación en las series de ficción.

\subsection{Objetivos e Hipótesis}

Nuestra investigación está basada en un estudio de caso sobre la serie de ficción "Las del Hockey", con una metodología mixta que tiene su fundamento en unos objetivos, entendidos como la meta que se pretende alcanzar con nuestro estudio y unas hipótesis que tienen como finalidad la declaración que, como investigadores, realizamos sobre el resultado de nuestra investigación. Estos objetivos son:

- Objetivo 1: Diagnosticar la evolución coeducativa de la ficción televisiva protagonizada por adolescentes.

- Objetivo 2: Descubrir aspectos y disruptivas de estereotipos de género discriminatorias de la serie "Las del Hockey".

- Objetivo 3: Analizar el contenido y el discurso coeducativo de la serie «Las del Hockey".

Como hipótesis presentamos las siguientes:

- Hipótesis 1: Existe una evolución coeducativa en el universo serial protagonizado por adolescentes.

- Hipótesis 2: Existen rupturas en los estereotipos y roles de género en la serie "Las del Hockey".

- Hipótesis 3: La serie de "Las del Hockey» es una serie coeducativa en las diferentes dimensiones laboral, interrelacional y personal.

\subsection{Instrumentos de investigación}

Los instrumentos utilizados en el estudio han sido las entrevistas semiestructuradas a una coautora y a ocho observadoras privilegiadas, entendiéndolas como "conocedoras expertas del fenómeno, con una visión directa y profunda del mismo que los sitúa en una posición de observación privilegiada" (Corbetta, 2003). Los perfiles son la cocreadora de la serie y experta en audiovisuales, Ona Anglada; y a expertas en el ámbito de la comunicación y la educación que han ejercido de audiencia. Este 
instrumento cualitativo se ha visto completado, desde una perspectiva de triangulación de resultados, con los datos recogidos del análisis de nueve perfiles de Instagram y del contenido con una escala de estimación de doce horas de producción de trece capítulos de la primera temporada. La audiencia la hemos analizado cuantitativamente comparándola con la primera y segunda temporada, para ello nos hemos servido del programa estadístico SPSS. El guión de las entrevistas y la escala de estimación han sido validadas por profesionales que forman parte del grupo GICID de la Universidad de Zaragoza (España). Las entrevistas han sido codificadas siguiendo el criterio presentado en la siguiente Tabla 1, mostrando también la especialidad y el ámbito laboral.

Tabla 1. Perfil de las personas entrevistadas

\begin{tabular}{|l|c|c|c|c|c|}
\hline Persona entrevistada & Código & Género & Edad & Especialidad & Ámbito Laboral \\
\hline Anglada, Ona & E-AON-01 & Femenino & 26 & $\begin{array}{c}\text { Coautora de la serie- } \\
\text { Audiovisuales }\end{array}$ & Universidad \\
\hline Álvarez M José & E-AMJ-02 & Femenino & 51 & Educación Física & Universidad \\
\hline Aleu, Cristina & E-ACR-03 & Femenino & 30 & Educación Física & Docencia \\
\hline Cantillo Ma Carmen & E-CMC-04 & Femenino & 58 & $\begin{array}{c}\text { Educación y Comuni- } \\
\text { cación }\end{array}$ & Universidad \\
\hline Delicado, María & E-DMA-05 & Femenino & 30 & $\begin{array}{c}\text { Gestión en adolescentes } \\
\text { Educación } \\
\text { Social }\end{array}$ \\
\hline Herrera, Sonia & E-HSO-06 & Femenino & 36 & Audiovisuales & Universidad \\
\hline Muñoz, Yolanda & E-MYO-07 & Femenino & 26 & Docencia & Docencia \\
\hline Ruíz, Noemi Patricia & E-RNP-08 & Femenino & 35 & Gestión social & $\begin{array}{c}\text { Educación } \\
\text { Social }\end{array}$ \\
\hline Higueras, Laura & E-HLA-09 & Femenino & 26 & Periodista Cultura-Tv & Periodismo \\
\hline
\end{tabular}

Fuente: Elaboración propia por los autores

Otro de los instrumentos utilizados es Test de Bechdel-Wallace, que permite observar la brecha de género en las producciones audiovisuales. Trata de una serie cuestiones que nacieron del cómic de Alison Bechdel "Dykes» (Bechdel, 1986). Las cuestiones son: $1^{a}$ ) ¿Aparecen dos mujeres en la película o serie?; $2^{a}$ ) ¿Hablan entre ellas?; y $3^{\text {a }}$ ¿La temática tratada va más allá del foco a un hombre? Si, tras ver el contenido, las y los espectadores responden «si» a las tres cuestiones, estaríamos hablando que superan Test de Bechdel, y nos mostrarían los resultados en función en términos de desigualdad de género, en "cumple» $\mathrm{O}$ «O no cumple». El hecho de que no aprueben una producción en particular no implica necesariamente que no haya participación femenina o no tenga contenido contra la igualdad. Para el análisis de contenido hemos creado una herramienta para medir el contenido coeducativo, cuya escala de estimación es 1 a 5 según se presenta en la Tabla 2. 
Tabla 2: Instrumento de medición coeducativa fuera del ámbito escolar

\begin{tabular}{|l|c|}
\hline \multicolumn{1}{|c|}{ Familias para el análisis coeducativo } & Parámetros de medición \\
\hline $\begin{array}{l}\text { Ámbitos personales: Sensibilización sobre igualdad, roles, } \\
\text { estereotipos; educación emocional y sexual; y representación } \\
\text { de la dicotomía entre ámbito público y privado respecto a los } \\
\text { espacios }\end{array}$ & De 1 a 5 atendiendo al parámetro \\
\hline $\begin{array}{l}\text { Ámbitos de relación e interrelación: Sororidad, lenguaje y comu- } \\
\text { nicación audiovisual no sexista, visibilidad de prácticas sexistas, } \\
\text { inclusión de la perspectiva de género y la teoría de género, y } \\
\text { sensibilización, prevención y lucha contra la violencia de género }\end{array}$ & De 1 a 5 atendiendo al parámetro \\
\hline $\begin{array}{l}\text { Ámbitos laborales: Reparto de responsabilidades domésticas, } \\
\text { conciliación de la vida personal y laboral; igualdad en la repre- } \\
\text { sentación laboral y académica }\end{array}$ & De 1 a 5 atendiendo al parámetro \\
\hline
\end{tabular}

Fuente: Elaboración propia por los autores

\section{Análisis de resultados}

La fase de análisis de resultados en un proceso de investigación donde se interpretan los datos recogidos es considerada como una etapa fundamental. A continuación, presentamos la información relevante tanto cualitativa como cuantitativa, acorde con los objetivos e hipótesis del estudio de caso planteado, manteniendo una interpretación fundamentada.

\subsection{Categoría 1: Series de ficción de coeducación}

El estudio pone de manifiesto el papel de los medios de comunicación y, por ende, de las series y películas como agente socializador de público adolescente, influyendo en la construcción de identidades y también en la permanencia o la ruptura de estereotipos de género (E-DMA-05). Las personas participantes en la muestra, como observadoras privilegiadas, coinciden en la necesidad de que exista una coeducación en estos espacios, partiendo de la demanda de la alfabetización digital (E-MYO-07) sobre "los instrumentos para analizar los medios, como por ejemplo aspectos del lenguaje visual como fondo, ropa, relación con un objeto.» (E-CMC-04). La coeducación se está proyectando en el ámbito de las series con mucha fuerza (E-CMC-04), «las series tienen un gran potencial en la coeducación» (E-MYO-07).

Estamos asistiendo a un aumento de las series de ficción por la mayor oferta de contenidos de las plataformas, enriqueciendo el universo de personajes, historias y tramas, y todo ello siendo protagonistas personas adolescentes. De forma paralela, observamos que nuestro entorno está cambiando y que, en estas series, también se presentan novedades sociales como «el feminismo (que) se hace explícito en la serie y el discurso ya responde a una traslación; no podríamos venir de los últimos 8 de marzo masivos a nivel mundial en la calle, en del "me too", qué sale a colación en el vídeo y de tantos movimientos que han tenido que ver con la discriminación y la violencia hacia las mujeres; eso sería como raro también que se obvie en la ficción televisiva». Por otra parte, esta dinámica de la ficción puede "normalizar y visibilizar realidades que ya existen o desear mundos posibles o mundos que podrían existir» (E-AON-01). Una vez más queda latente que «ninguna multinacional se doblega ante 
ningún movimiento social, más bien, lo que está sucediendo es que hay demanda de nuevas narrativas y de nuevos relatos (Mancho-Iglesia y Marta-Lazo, 2020). La ficción no podía quedarse atrás en los cambios que están sucediendo en la calle» (E-HSO-06). Por otra parte, la dinámica de la ficción puede «normalizar y visibilizar realidades que ya existen o desear mundos posibles o mundos que podrían existir» (E-AON-01).

Es importante resaltar la importancia para la etapa adolescente de la coeducación en series de ficción para que tenga una estrecha relación con una educación sexual y para la igualdad. Debemos tener en cuenta que mucha información llega de los medios, incluyendo de productos de pornografía (E-DMA-05), para ello es importante abogar por la perspectiva de género desde la naturalidad (E-RMP-08). Los resultados sobre de series de adolescentes reflejan una fuerte presencia de estereotipos y roles de género. En la Tabla 3 podemos ver las series más citadas por la muestra, emitidas a finales del siglo XX y observamos las referencias a su adolescencia como «Sensación de Vivir", "Compañeros» o "Física y Química». Gran parte de las tramas de estas series de ficción citadas no cumplen Test de Bechdel en la mayoría de sus episodios. Series como «Un paso adelante», pese a no ser protagonizada por adolescentes, se podía ver una ruptura de roles y estereotipos respecto la masculinidad y actividades como la danza o el baile (E-MYO-07); también veíamos una mayor normalización de las parejas del mismo género respecto hace 10 años (E-DMA-05.). En nuestra mirada a las series protagonizadas y, la mayoría de las veces, consumidas por adolescentes, resaltamos a "Buffy Cazavampiros", una serie sobre el empoderamiento de la mujer, con tratamientos de cuestiones de género y rompiendo estereotipos en diversas dimensiones como la emocional o la física, "Buffy fue una heroína que salvaba al mundo cada temporada, además se trataba de forma natural, por ejemplo, las relaciones lésbicas» (E-HSO-06).

El estudio nos permitió realizar también un diagnóstico sobre las series actuales en referencia a la adolescencia. Una de las producciones más citadas fue "Por 13 razones", serie con gran poder de concienciación en diferentes temáticas como el bullying o la violencia entre los jóvenes, que "ha puesto sobre la mesa temas no tratados en series de ficción como el bullying sexual, la violación o adolescencia” (E-HSO-06) y donde los guionistas recibieron fuertes críticas a la hora de abordar temas como el suicidio (E-DMA-05). Otra serie mencionada ha sido «Sex Education» que ha generado reflexiones en los adolescentes respecto a la igualdad de género y la diversidad sexual.

Hemos podido ver en esta categoría una evolución en materia de igualdad en relación con los contenidos de las series ficción consumidas por adolescentes, pero también hay series que sucumben a viejas fórmulas con nuevos formatos. Una de las series citadas fue "Élite», definida como "estereotipada, alejada de la realidad de los adolescentes en España», además, «reproduciendo estereotipos racistas» (E-HSO-06).

En definitiva, en las series de ficción se están rompiendo estereotipos, tan acuciados en el público adolescente, pero hay algunos que continúan muy arraigados como "Élite», que siguen manteniendo los roles de "chica buena" y "chico malo" (E-MYO-7), repitiendo estereotipos como en la tradicional serie de "Compañeros» (E-HSO-06). En esta línea de contenidos estereotipados podemos observar en producciones actuales (2020) protagonizas por adolescentes papeles como "Eleven» (Stranger Things), donde la heroína salva el mundo rompiendo los estereotipos (E-ACR-3). Varias entrevistadas mostraron una predisposición a utilizar la serie de nuestro estudio en el campo de la docencia como herramienta educativa y transversal, resaltado su importancia como valor pedagógico, convirtiendo nuestro estudio en un proceso de transferencia del conocimiento con un símil de investigación-acción. Respecto a ello, el 
profesorado participante «utilizaría fragmentos algún capitulo sacando trozos específicos o eligiendo algún capítulo que fuese importante, dependiendo del bloque de contenidos que vaya a trabajar, sé que hay profesoras que lo han hecho sobre esta serie, en su ámbito, en la educación física» (E-AMJ-02), ofreciendo así a la sociedad un respiro coeducativo.

En las Tablas 3 y 4 podemos observar las series de adolescencia en relación con el instrumento diseñado para los diferentes apartados de la muestra, donde podemos ver una diferencia significativa de en la evolución de las series. También hemos incluido el Test de Bechdel, donde "Las del Hockey» la cumple al realizarse conversaciones de algo que no sea un hombre. Este test se ha generalizado en la industria del cine para analizar la presencia femenina en películas y series.

Tabla 3. Diagnóstico de las series de adolescentes proyectadas en la muestra

\begin{tabular}{|l|c|c|c|c|c|c|}
\hline \multicolumn{1}{|c|}{ Nombre } & Citada & Emisión & Personal & Relacional & Laboral & $\begin{array}{c}\text { Test } \\
\text { Bechdel }\end{array}$ \\
\hline Sensación de Vivir & 3 & $1990-2000$ & 3 & 3 & 4 & No cumple \\
\hline Física y Química & 3 & $2008-2011$ & 3 & 2 & 3 & No cumple \\
\hline Compañeros & 2 & $2007-2013$ & 3 & 3 & 3 & No cumple \\
\hline
\end{tabular}

Otras series citadas: Freaks \& Geeks, Skam, Verano Azul, Compañeros, El Internado, Upa Dance, El príncipe de Bel Air, Al Salir de clase, Buffy: La cazavampiros

\begin{tabular}{|l|c|c|c|c|c|c|}
\hline Sex Education & 4 & $2019-2020$ & 5 & 5 & 4 & Cumple \\
\hline Por 13 razones (Netflix) & 5 & $2017-2020$ & 4 & 3 & 3 & Cumple \\
\hline Élite & 6 & $2018-2020$ & 2 & 1 & 2 & No cumple \\
\hline
\end{tabular}

Otras series citadas: Merlí (TV3/Netflix), Stranger Things (Netflix), Creedme (Netflix), Never Have I Ever (Netflix), La otra mirada, Las aventuras terroríficas de Sabrina

Fuente: elaboración propia por los autores

Tabla 4: Instrumento de medición coeducativa fuera del ámbito escolar

\begin{tabular}{|l|l|}
\hline \multicolumn{1}{|c|}{ Familias para el análisis coeducativo } & Nota media \\
\hline $\begin{array}{l}\text { Ámbitos personales: Sensibilización sobre igualdad, roles, estereotipos; educación } \\
\text { emocional y sexual; y representación de la dicotomía entre ámbito público y privado } \\
\text { respecto a los espacios }\end{array}$ & $4,8 / 5$ \\
\hline $\begin{array}{l}\text { Ámbitos de relación e interrelación: Sororidad, lenguaje y comunicación audiovisual } \\
\text { no sexista, visibilidad de prácticas sexistas, inclusión de la perspectiva de género y la } \\
\text { teoría de género, y sensibilización, prevención y lucha contra la violencia de género }\end{array}$ & $4,4 / 5$ \\
\hline $\begin{array}{l}\text { Ámbitos laborales: Reparto de responsabilidades domésticas, conciliación de la vida } \\
\text { personal y laboral; igualdad en la representación laboral y académica }\end{array}$ & $4,33 / 5$ \\
\hline
\end{tabular}

Fuente: elaboración propia por los autores 


\subsection{Categoría 2: Cultura participativa}

\subsubsection{Subcategoría: Impacto social en audiencia}

Los capítulos de la serie "Las del Hockey" con mayor audiencia, como observamos en la Tabla 5, han sido emitidos en Tv3. Podemos constatar que los episodios de la segunda temporada protagonizan esta clasificación, pero, no obstante, el primer puesto es para el capítulo "Emma" de la primera temporada, con 450.000 televidentes. Observamos que la media por episodio, que formaron parte de la cultura participativa desde el papel de audiencia, ha aumentado en la segunda temporada (356.600) respecto a la primera (290.530); la media de la segunda ha aumentado en 62.000, según se presenta en la Tabla 6. Por el periodo de emisión, podemos relacionar el incremento de audiencia al confinamiento durante la pandemia provocada por la Covid-19, pero también por la influencia que pueda generar el estreno de la primera temporada en los contenidos de la plataforma digital de Netflix en septiembre de 2019. En cuanto a la media de cuota de pantalla en Cataluña, observamos una línea ascendente de la segunda temporada $(13,57)$ respecto a la primera $(11,4)$.

Las emisiones fueron los lunes a las 21: 55 en el canal autonómico Tv3, siendo una hora idónea para poder verla en familia, y a su vez, teniendo en cuenta la ausencia de las clases presenciales y la promoción del teletrabajo para los días posteriores debido al periodo de confinamiento. Podemos comprobar un aumento en más de dos puntos entre ambas temporadas.

El deporte tiene un gran impacto en la audiencia, temática de la serie de ficción, pero la gran parte de las emisiones deportivas son el fútbol, llegando a ser un $90 \%$ de las retransmisiones (E-AON-01). De ese porcentaje, el 99\% podría hacer referencia al fútbol masculino. En esa línea, la muestra de nuestro estudio manifiesta (E-MYO-07) que el deporte femenino está infravalorado en los medios de comunicación, y cuando se emite, se retrasmiten en canales de escasa audiencia. Es importante «una mayor presencia del deporte femenino en los medios, pudiendo cambiar positivamente los referentes adolescentes" (E-ACR-03). La presencia de series de ficción corales, con contextos de equipos deportivos, permite reivindicar diversos papeles y diferentes realidades (E-DMA-05). Las plataformas como Netflix buscan un feedback con el público en diversos nichos, como apunta «muchas veces lo que sale no es toda una imagen para romper lo establecido sino también lo que el público pide» (E-CMC-04). Esta demanda permite crear nichos con contenidos muy concretos, y a su vez se crea una presión para que en el sector audiovisual se produzcan cambios positivos (E-AON-01).

Tabla 5. Datos de los 10 capítulos con máxima audiencia emitidos por TV3 en Cataluña $\left(1^{\mathrm{a}}\right.$ y $2^{\mathrm{a}}$ temporada).

\begin{tabular}{|l|c|c|c|c|c|}
\hline & Capítulo & Temporada & Fecha & Espectadores & Cuota \\
\hline 1. & $1^{\circ}$ «Emma" & Primera & 29 de abril de 2019 & 450.000 & 15,6 \\
\hline 2. & $14^{\circ}$ "Tot canura" & Segunda & 30 de marzo de 2020 & 450.000 & 16,2 \\
\hline 3. & $20^{\circ}$ "Caure" & Segunda & 11 de mayo de 2020 & 375.000 & 14,5 \\
\hline 4. & $17^{\circ}$ "Secrets» & Segunda & 20 de abril de 2020 & 367.000 & 14,2 \\
\hline 5. & $18^{\circ}$ "La fugida" & Segunda & 27 de abril de 2020 & 366.000 & 13,5 \\
\hline
\end{tabular}


JAVIER GIL-QUINTANA Y SIMÓN GIL-TÉVAR

SERIES DE FICCIÓN COMO MEDIO DE COEDUCACIÓN PARA ADOLESCENTES. ESTUDIO DE CASO: LAS DEL HOCKEY

\begin{tabular}{|l|c|c|c|c|c|}
\hline 6. & $21^{\circ}$ "Guanyar o perdre» & Segunda & 18 de mayo de 2020 & 361.000 & 13,3 \\
\hline 7. & $6^{\circ}$ "Flor» & Primera & 3 de junio de 2019 & 356.000 & 13,4 \\
\hline 8. & $25^{\circ}$ "Ruptures» & Segunda & 15 de junio de 2020 & 353.000 & 14 \\
\hline 9. & $24^{\circ}$ "Sempre endavant" & Segunda & 8 de junio de 2020 & 352.000 & 14,5 \\
\hline 10. & $19^{\circ}$ "La traïció» & Segunda & 4 de mayo de 2020 & 351.000 & 13,00 \\
\hline
\end{tabular}

Fuente: elaboración propia por los autores

Tabla 6. Media de espectadores y cuota de la $1^{a}$ y $2^{a}$ temporada en TV3

\begin{tabular}{|l|c|c|}
\hline \multicolumn{1}{|c|}{ Temporada } & Media de espectadores & Media de cuota \\
\hline Primera & 290.530 & 11,4 \\
\hline Segunda & 352.600 & 13,57 \\
\hline
\end{tabular}

Fuente: elaboración propia por los autores

\subsubsection{Subcategoría: Redes de influencers}

Durante la emisión de la primera temporada en TV3, se crearon unos perfiles corporativos de las siete jugadoras en Instagram, fomentando, en base a la cultura participativa, una red de influencers. Gracias a la coralidad de la serie, en la primera temporada se dedican los capítulos a las protagonistas, siendo acompañadas por las cuentas de Instagram en los rótulos de inicio, red social más seguida por el público adolescente. Con ello, se incorporaban debates en Twitter y sobre todo en Instagram (E-AON-01) fomentando así la generación de un discurso transmedia en torno a la serie, sus tramas y protagonistas. Se compartía contenido, a propósito de lo que había pasado en el capítulo anterior o de lo que podía pasar, «era muy interesante ver cómo los fans comentaban y daban su opinión sobre diversos temas» (E-AON-01). En el espacio de tiempo en que se emitió la producción sobre la relación no consentida, «se realizó un post preguntando 'sí Óscar había hecho algo mal; una pregunta que abría el debate', y había muchísima gente en el debate 'si lo ha hecho muy mal', sí las mismas fans y adolescentes hablaban de este tema, y aún no había una posición muy clara de condenarlo, de que en seguida viesen que estaba mal, o había muchos matices»( E-AON-01), fomentando así la cultura participativa de la audiencia y, claramente, la influencia en la ideología del público adolescente. Otra temática que generó bastante debate fueron las relaciones abiertas, y el nivel de compromiso desigual en la relación de Lorena y Gina. La cultura participativa se proyectó en estas redes de influencers a través de los debates en social media, fomentando la reflexión y la participación, dejando atrás a un consumidor pasivo que visiona la serie (E-MYO-07). El público tiende a discernir ligeramente entre actores/actrices los personajes de la serie, "creemos que son reales, que las personas son así" (E-ACR-03). Como se presenta en la Tabla 7 , los perfiles en redes sociales de los actores han superado a los propios personajes de las cuentas corporativas de la serie, destacando Claudia Riera y Mireia Orio como las actrices con más influencia. La muestra de observadoras privilegiadas recuerda la importancia que, en la etapa de la adolescencia, tengan las series un referente de su edad, "que les hable de las mismas vivencias que están viviendo" (E-ACR-03). La influencia y la repercusión mediática de los actores y actrices permiten trasladar los debates 
en las diferentes cuentas, convirtiéndose en los mejores referentes e influencers de la propia marca de la serie.

Tabla 7. Análisis de cuentas de Instagram en relación con su impacto.

\begin{tabular}{|c|c|c|c|c|c|c|}
\hline Papel & Actor & Cuenta personal & $15-6-20$ & $1-8-2020$ & Cuenta corporativa & $1-8-20$ \\
\hline Emma & Délia Brufau & @deliabrufau & $30 \mathrm{mil}$ & $30 ’ 8 \mathrm{mil}$ & 3mma_ricou & 6.210 \\
\hline Anna & Iria del Rio & @iriadelrioo & $46 \mathrm{mil}$ & $48 ’ 2$ mil & - & - \\
\hline Berta & Natália Barrientos & @natbarrientosb & - & $26 ` 6 \mathrm{mil}$ & @bertafterrats & 4.768 \\
\hline Lorena & Mireia Orio & @mireiiaoriol & $73 \mathrm{mil}$ & $75 ' 8 \mathrm{mil}$ & @lorelai777 & 5.695 \\
\hline Raquel & Júlia Gilbert & @juliagibert & $29 \mathrm{mil}$ & $29^{\prime} 8 \mathrm{mil}$ & @rachel_alcober & 5.840 \\
\hline Flor & Asia Ortega & @asoaortega & $39 \mathrm{mil}$ & $41^{\prime} 2 \mathrm{mil}$ & @hockeyflower & 6.077 \\
\hline Gina & Claudia Riera & @cloriera & $50 \mathrm{mil}$ & $97^{\prime} 8 \mathrm{mil}$ & @va_6ina & 5.014 \\
\hline Laíla & Yasmina & @yasminadrissi & $18 \mathrm{mil}$ & $18^{\prime} 8 \mathrm{mil}$ & @sailorlaila & 4.754 \\
\hline Germán & Marc Clotet & @marc_clotet & $150 \mathrm{mil}$ & $154 \mathrm{mil}$ & - & - \\
\hline
\end{tabular}

Fuente: Elaborada por los autores

Observamos el aumento considerable en miles de seguidoras y seguidores de la actriz Claudia Riera, del 15 de junio al 1 de septiembre del 2020, incrementándose en 95,6 \%, como se observa en la Tabla 7 y en el Gráfico 1. Gran parte de ello guarda relación con su presencia en el spin-off «Vis a Vis: Oasis» (2020); aun así, podemos ver un incremento en todos los perfiles de las actrices en 1,6\%, sin incluir a la misma actriz y al actor Marc Clotet (Germán). Aunque Iría del Rio como Marc Clotet no tuvieran cuentas corporativas como las protagonistas ambos llegan a los 30 años y tienen un mayor repunte e impacto en redes. Otra observación que podemos ver es que, tanto Natália Barrientos como Yasmina, tienen menos seguidoras y seguidores, siendo perfiles más diversos y de ruptura con los estereotipos físicos y étnicos.

Gráfico 1. Número de impacto de los personajes/actrices en seguidores en miles en Instagram

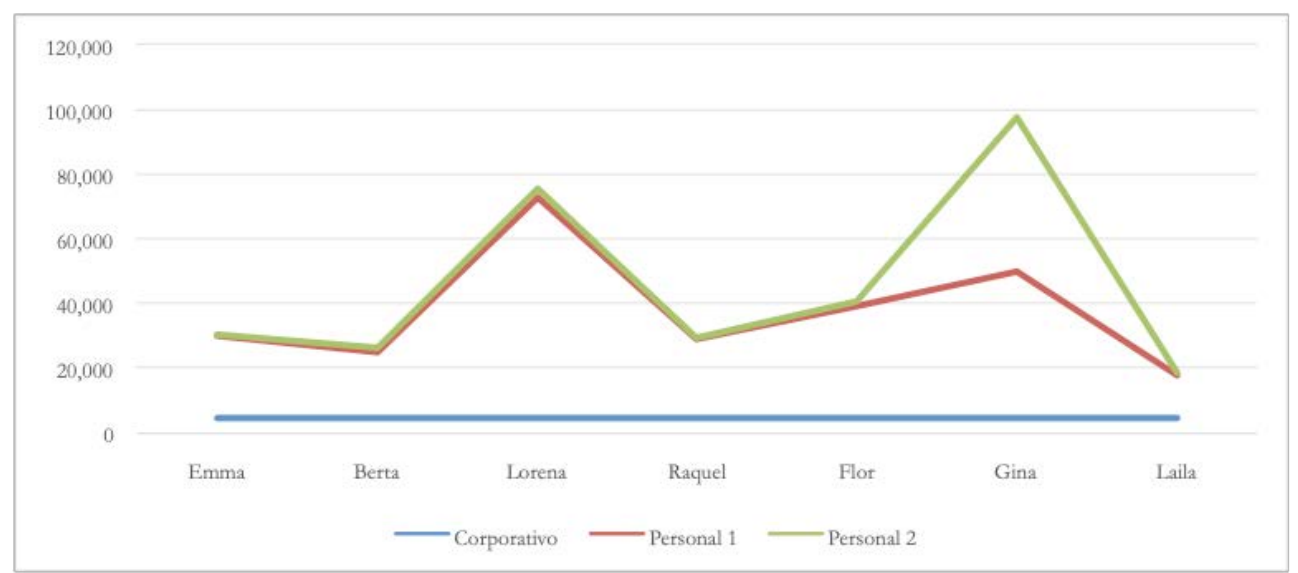

Fuente: Instagram. Número de impacto de personaje/actrices 


\subsection{Categoría 3: Estereotipos personales, relacional y laborales}

\subsubsection{Subcategoría: Estereotipos personales}

El análisis de la información que nos refleja el estudio arroja resultados positivos respecto el tratamiento de la serie "Las del Hockey» en la ruptura de estereotipos en el ámbito personal. Los datos reflejados nos muestran una cifra, nada desdeñable, de una media de 4`68 de estimación coeducativa sobre 5; hablamos de un valor de 93,6\% en el contenido sobre estereotipos y aspectos personales, como se observa en la Tabla 8. En nuestro panorama televisivo estamos acostumbrados a ver mujeres estereotipadas bajo un canon de aspecto físico de belleza y de comportamiento pasivo, una ficción androcentrista romántica ligada a la representatividad de «una mujer 'cheerleader' americana, muy alejada de nuestras experiencias» (E-AON-01). A pesar de ello cada vez son más series que tratan una diversidad de mujeres, "con perfiles menos idealizados y diferentes entre ellas, tanto físicamente como de personalidad» (E-HLA-09). Unos de los más comunes que podemos observar es la representación de la mujer en el deporte, donde existe una imagen hipersexualizada o silenciada. Las personas participantes, como observadoras privilegiadas, coinciden que "Las del Hockey" rompen con características sexistas: "podemos ver 'chicas haciendo deporte real, jugando, sufriendo, divirtiéndose, jugando contra chicos' "(E-ACR-03). La serie resalta la importancia de darles protagonismo a mujeres de diferentes edades con la ropa adecuada a la escena. Por ejemplo, en la serie se pueden observar prendas deportivas sin tintes eróticos (E-DMA-05). En esta misma línea (E-ACR-03) añade «que podemos ver chicas en chándal, chicas que se quitan los pendientes para hacer deporte».

Observamos como el sexo y la mujer siempre ha sido un tema tabú, y sigue siendo un tema recurrente en las series de adolescentes la perdida de la virginidad de la mujer, siendo diferente entre chicos y chicas (E-ACR-03). También en esta línea es necesario resaltar la visibilización del anticonceptivo masculino (preservativo) en la serie "Las del Hockey», alejados de los arrebatos tan vistos en la ficción (E-AON01). En los datos que ofrece la Tabla 8 podemos encontrar diversidad de situaciones, no existiendo una frecuente dicotomización de espacio en relación hombre-público, mujer-privado. Podemos ver mujeres y hombres en los diferentes espacios comunes, tanto en el hogar como el trabajo rompiendo el común «reflejo de la mujer en el ámbito privado y lo íntimo, en la casa y el hombre en el exterior, en lo público. Están ahí eso esos roles que reproducen la sociedad” (E-CMC-04).

Tabla 8. Análisis coeducativo sobre aspectos personales

\begin{tabular}{|c|c|c|c|c|c|c|c|}
\hline \multicolumn{6}{|c|}{ Aspectos personales } & $\begin{array}{l}\text { Valor total: } \\
4.68\end{array}$ & Valor \%: $93.6 \%$ \\
\hline \multicolumn{6}{|c|}{ Sensibilización sobre igualdad, roles, estereotipos } & \multicolumn{2}{|c|}{ Valor: 4.4} \\
\hline $\begin{array}{l}\text { Ausencia de competitividad } \\
\text { (mujer) }\end{array}$ & 1 & 2 & 3 & 4 & 5 & \multicolumn{2}{|c|}{$\begin{array}{l}\text { Ausencia de estereotipos en } \\
\text { relación con la competición }\end{array}$} \\
\hline $\begin{array}{l}\text { Ausencia de expresión } \\
\text { afectiva } \\
\text { (hombre) }\end{array}$ & 1 & 2 & 3 & 4 & 5 & \multicolumn{2}{|c|}{$\begin{array}{l}\text { Ausencia de estereotipos en } \\
\text { relación con la expresión } \\
\text { afectiva }\end{array}$} \\
\hline
\end{tabular}




\begin{tabular}{|c|c|c|c|c|c|c|}
\hline $\begin{array}{l}\text { Liderazgo bajo el rol mascu- } \\
\text { lino }\end{array}$ & 1 & 2 & 3 & 4 & 5 & Liderazgo sin estereotipos \\
\hline Indumentaria sexualizada & 1 & 2 & 3 & 4 & 5 & $\begin{array}{l}\text { Indumentaria ajustada al } \\
\text { contexto }\end{array}$ \\
\hline Mujer/delgada, hombre/fuerte & 1 & 2 & 3 & 4 & 5 & $\begin{array}{l}\text { Riqueza en la diversidad de } \\
\text { papeles }\end{array}$ \\
\hline \multicolumn{6}{|c|}{ Educación emocional y sexual } & Valor: 4.66 \\
\hline $\begin{array}{l}\text { Actitud pasiva/activa en } \\
\text { el sexo en relación mujer/ } \\
\text { hombre }\end{array}$ & 1 & 2 & 3 & 4 & 5 & Actitudes diversas \\
\hline Modelo heteropatriarcal & 1 & 2 & 3 & 4 & 5 & Diversidad sexual \\
\hline $\begin{array}{l}\text { La sexualidad de la mujer es } \\
\text { tabú }\end{array}$ & 1 & 2 & 3 & 4 & 5 & Naturalidad en el sexo \\
\hline \multicolumn{6}{|c|}{$\begin{array}{c}\text { Representación de la dicotomía entre ámbito público y privado } \\
\text { respecto a los espacios }\end{array}$} & Valor: 5 \\
\hline $\begin{array}{l}\text { Masculino-público/ femenino- } \\
\text { privado }\end{array}$ & 1 & 2 & 3 & 4 & 5 & $\begin{array}{l}\text { Espacios comunes (hogar, } \\
\text { trabajo y ocio) }\end{array}$ \\
\hline
\end{tabular}

Fuente: elaboración propia por los autores

\subsubsection{Subcategoría: Sororidad, estereotipos e interrelaciones}

Un concepto muy importante tratado en la serie es la sororidad. Existe bastante unanimidad sobre la esencia del término en diferentes tramas de la serie, pero más concretamente en la escena de la producción 2 protagonizada por Berta y Raquel. Destaca la ruptura del estereotipo de la rivalidad entre mujeres que ofrecen otras series, mostrándose mutuamente un apoyo, y empatizando sobre sus sensaciones (E-MYO-07). La escena «el estereotipo de la rivalidad entre las mujeres, que se llevan muchísimo en todas las películas, desaparece» (E-CMC-04); la intencionalidad era que la sororidad «estuviese siempre presente en el mensaje final de la serie, y qué manchase cada escena, cada trama, cada momento como la guía» (E-AON-01). El valor del análisis subscribe a las personas participantes en la muestra obteniendo la máxima nota (5) que mide el propio concepto presentado en la Tabla 9.

Otra de las temáticas importantes a mencionar es sobre la violencia sobre la mujer (producción 2). abusos, violaciones o relaciones no consentidas son una realidad silenciada que sufre la mujer. Hay bastante unanimidad entre las personas observadoras privilegiadas en destacar positivamente la visibilización e identificación de este tipo de violencia o abusos sexuales que se puede dar en la pareja. También resaltan la ausencia de esta temática en gran parte de los productos audiovisuales. La muestra denuncia que «se han empeñado en dibujarnos al violador de pasamontañas, callejón oscuro y cuchillo, y no es lo habitual. Se produce muchas más agresiones en ámbitos de confianza, espacios de ocio y nos deja más desarmadas, porque, en nuestro imaginario no está» (E-HSO-06). La serie también trabaja desde una perspectiva de género y de forma crítica el slutshaming (tildar de prostituta), fat shaming (insultar por sobrepeso), aborto en una menor y el ciberacoso. La propuesta en los diferentes apartados de la Tabla 9 ponen en relevancia que el aspecto coeducativo y feminista impregna todas las tramas. 
El término "guerra de sexos" ha sido reflejado en numerosas ocasiones en las múltiples pantallas, sobre todo en el ámbito de la comedia. El concepto fue introducido en los datos recogidos en el estudio para hablar sobre la producción 3, que trataba sobre un partido amistoso entre chicas y chicos para solucionar el uso de los vestuarios. Tras la victoria del equipo femenino, la directiva muestra su negativa para dar preferencia a la sección masculina, pero los chicos optan por dar la cara por sus compañeras ante las reticencias del presidente. El concepto guerra de sexos «está un poco desfasado, pero se sigue fomentando y presentando como una cuestión de lucha de poder» (E-HSO-06). La muestra reseña positivamente el partido como una forma de rivalidad deportiva, "tratándose en igualdad, y sabiendo rivalizar en el ámbito deportivo» (E-RNP-08).

Tabla 9. Análisis coeducativo sobre aspectos de relaciones e interrelaciones

\begin{tabular}{|c|c|c|c|c|c|c|c|}
\hline \multicolumn{6}{|c|}{ Relaciones e interrelaciones } & Valor total: 4.4 & Valor \%: $88 \%$ \\
\hline \multicolumn{6}{|c|}{ Sororidad } & \multicolumn{2}{|c|}{ Valor medio: 5} \\
\hline Rivalidad entre mujeres & 1 & 2 & 3 & 4 & 5 & Sororidad & \\
\hline \multicolumn{6}{|c|}{ Sensibilización, prevención y lucha contra la violencia de género. } & \multicolumn{2}{|c|}{ Valor medio: 4} \\
\hline Violencia implícita & 1 & 2 & 3 & 4 & 5 & Prevención y se & sibilización \\
\hline \multicolumn{6}{|c|}{ Lenguaje y comunicación audiovisual no sexista. } & \multicolumn{2}{|c|}{ Valor medio: 4} \\
\hline $\begin{array}{l}\text { Lenguaje sexista y discrimi- } \\
\text { natorio }\end{array}$ & 1 & 2 & 3 & 4 & 5 & Lenguaje inclus & o y no sexista \\
\hline \multicolumn{6}{|c|}{ Inclusión de la perspectiva de género y la teoría de género. } & \multicolumn{2}{|c|}{ Valor medio: 5} \\
\hline Posición machista & 1 & 2 & 3 & 4 & 5 & \multicolumn{2}{|c|}{ Perspectiva feminista } \\
\hline \multicolumn{6}{|c|}{ Visibilidad de prácticas sexistas. } & \multicolumn{2}{|c|}{ Valor medio: 4} \\
\hline Prácticas sexistas & 1 & 2 & 3 & 4 & 5 & Ausencia de sex & smo \\
\hline Guerra entre sexos & 1 & 2 & 3 & 4 & 5 & Ausencia & \\
\hline
\end{tabular}

Fuente: elaboración propia por los autores

\subsubsection{Subcategoría: Estereotipos laborales}

El análisis respecto a los estereotipos y aspectos laborales comprenden la conciliación la laboral y la situación académica de la mujer representada en la serie, datos presentados en la Tabla 10. En el primer aspecto tratado, se muestra la conciliación laboral (estimación 4 sobre 5); tanto la mujer como el hombre concilian trabajo y familia en la serie, pero también es importante añadir la ausencia de niños y niñas en la serie. Respecto a los otros puntos sobre la "situación laboral y académica de la mujer representada en la serie», la estimación de valorización es muy significativo con un 4,66 (sobre 5). Sin duda un reflejo de la representación de género equitativa y variada. Dentro del universo de ficción, tanto mujeres como hombres desempeñan trabajos cualificados y remunerados, como cualificados y no remunerados. En la serie podemos ver un matrimonio como socios en una empresa, una abogada de éxito o la 
entrenadora de la sección masculina (E-RMP-08). Como hemos comentado anteriormente, las series de ficción es una herramienta de socialización que permite reflejar una realidad como si fuera un valor en sí mismo, creando imaginarios que "puedan reflejar cambios en la realidad"(E-HSO-06). Respecto a ello, la serie reivindica de la mujer en puestos directivos, y «enmarca la necesidad de una mayor conciliación laboral, e igualdad de género" (E-DMA-05).

En el último vídeo presentado en el estudio se muestra el ofrecimiento para el puesto de coordinación a la entrenadora de la sección femenina. La propuesta viene de la entrenadora del equipo masculino y aspirante a la presidencia del Club. En el vídeo «muestra a dos mujeres capaces de enfrentarse a cualquier puesto directivo, y buscan entre ellas cierto grado de complicidad (sororidad)» (E-AMJ-02). En el mismo fragmento de video también podemos ver como a Anna le entran dudas sobre si es capaz, en este sentido la muestra señala que, cuando las mujeres acceden o se les ofrece cargos superiores, se pone en duda tanto su valía, y su forma de llegar. En esta línea, (E-CMC-04), subraya que existen críticas "malpensadas» y si dan el poder es por tener alguna relación sentimental o familiar con un hombre, que el que ostenta el poder.

La mayor presencia de la mujer en los ámbitos profesionales y sociales va a favorecer un punto de vista más real de la perspectiva de género en las producciones de ficción. Para (E-HLA-09), «la ascensión laboral es lenta, pero poco a poco se va accediendo a puestos de poder, 'traducido a todos los ámbitos, eso también se está reflejando más en la ficción’”. Un ejemplo de ello lo podemos ver en la creación de la serie que hemos investigado, creada por Ona Anglada, Laura Azemar, Natalia Boadas y Marta Vivet o otras series de éxito como el "Orange is the new black" de Jenji Kohan.

Tabla 10. Análisis coeducativo sobre aspectos laborales

\begin{tabular}{|c|c|c|c|c|c|c|c|}
\hline \multicolumn{6}{|c|}{ Aspectos laborales } & Valor total: 4.33 & Valor $\%: 86 \%$ \\
\hline \multicolumn{6}{|l|}{ Conciliación laboral } & \multicolumn{2}{|c|}{ Valor: 4} \\
\hline No hay conciliación laboral & 1 & 2 & 3 & 4 & 5 & $\begin{array}{l}\text { La mujer y el hor } \\
\text { trabajo y familia }\end{array}$ & bre concilian \\
\hline \multicolumn{6}{|c|}{ Situación laboral y académica de la mujer representada en la serie } & \multicolumn{2}{|c|}{ Valor: 4,66 } \\
\hline $\begin{array}{l}\text { Trabajos domésticos no } \\
\text { remunerados }\end{array}$ & 1 & 2 & 3 & 4 & 5 & \multicolumn{2}{|c|}{ Trabajos remunerados } \\
\hline La mujer no tiene estudios & 1 & 2 & 3 & 4 & 5 & \multicolumn{2}{|c|}{$\begin{array}{l}\text { La mujer tiene formación } \\
\text { académica }\end{array}$} \\
\hline $\begin{array}{l}\text { La mujer tiene un puesto } \\
\text { laboral de subordinación }\end{array}$ & 1 & 2 & 3 & 4 & 5 & \multicolumn{2}{|c|}{$\begin{array}{l}\text { La mujer ostenta una alta } \\
\text { posición laboral }\end{array}$} \\
\hline
\end{tabular}

Fuente: elaboración propia por los autores

\section{Discusión}

La finalidad de un estudio de caso es la particularización, no tanto su generalización (Stake, 2010). Partiendo de esta afirmación, en nuestra investigación hemos tomado el caso particular de la serie "Las del Hockey» y hemos pretendido conocer su 
proyección, teniendo como anteojo la coeducación, la intención y grado de impacto en las mentes de los consumidores adolescentes. Destacamos en el estudio de corte mixto la unicidad, al haber indagado en otros estudios realizados sobre distintas producciones mediáticas y que nos han servido de cimiento para mejorar la comprensión de nuestro caso. En base a los objetivos e hipótesis planteadas en el estudio de caso, hemos podido comprobar que estos elementos del proceso de investigación han sido confirmados, otorgando así una validez clara a nuestro estudio y una consolidación a aquellos interrogantes que, como investigadores, nos planteamos en el proceso inicial.

Las series de ficción se han convertido en un agente de socialización para el público adolescente; la sociedad cambia y estas series manifiestan también estos cambios. Estas producciones tienen un gran potencial para la coeducación, aunque no todos los planteamientos de personajes, historias y tramas responden a esta perspectiva. Algunos trabajos previos también resaltan el poder de la "mass media" como fin educativo y para contrarrestar estereotipos de género, pudiendo moldear comportamientos y modificar actitudes sexistas (Belmonte y Guillamón, 2008; Galán-Fajardo, 2006; García y Calleja, 2006). Es importante resaltar, que además de la influencia de las series para poder llegar a una transformación social, se necesita una intervención en diferentes ámbitos como la educativa o institucional (Perelló, 2019). A finales del siglo XX las series consumidas y protagonizadas por adolescentes como «Buffy Cazavampiros" O "Skins", rompieron con roles y estereotipos tradicionales y contrarios a la igualdad de oportunidades, abriendo paso al empoderamiento de la mujer, el respeto a las relaciones entre personas del mismo sexo, etc.; en esta línea de pensamiento no se proyectaban otras series como "Compañeros» o "Física y Química ", donde por ejemplo, en esta última presentaban acciones estereotipadas y prevalecían comentarios sexualizados sobre la mujer (Galeón., 2019).

Las series de adolescentes emitidas en esta etapa del siglo XXI en que nos encontramos presentan una fuerte influencia de estereotipos y roles de género. Series como "Por 13 razones" trabaja temas sobre el bullyng, suicidio, pero también sobre el machismo de la sociedad y la violencia sobre la protagonista (Hernández-Carrillo, 2017); "Sex Education" ofrece un amplio abanico de posibilidades para educar en la igualdad y el respeto a la diversidad sexual. Diversos estudios han señalado la importancia que tienen estas series al tratar temáticas silenciadas con un discurso crítico y reflexivo (Peréz, 2019). Dentro del marco español podemos encontrarnos a series adolescentes como "La otra mirada» $\mathrm{O}$ "Las del Hockey», o no adolescentes como "Las chicas del cable» (Campos, 2017-2020) que forman parte de una segunda etapa de series corales (2014-2019), caracterizadas por la coincidencia con cuarta ola del feminismo, y con un guión que profundiza en temáticas relacionadas con la mujer y sus derechos (Ruíz y Pérez-Rufi, 2020). En esta etapa, que hemos incluido nuestra serie tratada, destacamos temáticas como el empoderamiento femenino, maternidad y conciliación laboral, la virginidad, el silenciado deporte femenino, la naturalidad en las relaciones LGTB, la sororidad; y, por supuesto, denunciando situaciones como el abuso dentro de las relaciones, la posesión-sumisión en las relaciones, bullying o ciberacoso. Otras series tratan temáticas novedosas en la pantalla como «Merlí», tratando la transexualidad o la naturalización de relaciones homosexuales, aunque recae en viejos estereotipos de género y en la narración de una historia como es abusos en redes, donde la mirada y el foco recae en la víctima y no en el agresor (Bonavitta y De Garay, 2019). Pero aún siguen emitiéndose otras producciones como "Élite» donde el lenguaje 
y la trama tiene un tinte menos avanzado a nivel de pensamiento y más estancado a nivel de promoción de la igualdad.

"Las del Hockey» respecto a las personas que formaron parte de la cultura participativa desde el papel de audiencia, ha aumentado en la segunda temporada (356.600) sobre la primera (290.530), aumentando en 62.000. Esta producción presenta varios puntos de reflexión de ruptura sobre los contenidos sobre estereotipos y aspectos personales, pero también realiza una denuncia social como la discriminación en el deporte. La mujer sigue siendo hipersexualizada o silenciada en el ámbito deportivo y gran parte de las producciones deportivas en el ámbito de ficción son protagonizadas por papeles masculinos, mientras que los papeles protagonistas de las mujeres oscilaban en un 18.60 \% (Ramírez-Macías, et. alt., 2011). Esta serie se presenta disruptiva con los roles protagonistas y las características sexistas, incluso en detalles tan simples, pero a la vez tan significantes, como el abordaje tendencioso de los adultos en la disputa por los vestuarios. Podemos ver mujeres y hombres en espacios comunes, tanto en el hogar familiar como en el laboral rompiendo el común reflejo del sexo femenino destinado al ámbito privado y lo íntimo y el hombre en el exterior, en lo público. Una ruptura con una dicotomía hombre-mujer que suele ser común en espacios y actividades en las ficciones o publicidad. (Belmonte y Guillamón, 2008; Verdú, 2016). La presencia de series como «Las del Hockey» en contextos deportivos permite reivindicar diversos papeles y diferentes realidades.

El concepto sororidad se encuentra presente en la serie, una palabra que habla de solidaridad, hermandad, complicidad y alianza entre las mujeres. La intención del planteamiento de la serie era que la sororidad estuviese siempre presente en el mensaje final y qué manchase cada escena, cada trama, cada momento como la guía. En esta línea, otros estudios coinciden en resaltar la importancia de este concepto en la lucha por los derechos de la mujer (Ruíz y Pérez-Rufi, 2019). Esta unión feminista se hace imprescindible en un contexto donde aumenta cada año la violencia machista o los abusos sexuales y que, las distintas producciones, silencian. El violador se presenta en el imaginario social como aquel hombre de pasamontañas, en un callejón oscuro y con un arma blanca, pero esto no es lo habitual. Las agresiones se llevan a cabo en ámbitos de confianza, espacios de ocio y deja a las mujeres desarmadas, porque, en nuestro imaginario creado por las producciones de ficción, no está esa situación. La serie "Las del Hockey" trabaja todas estas problemáticas desde una perspectiva de género y tacha de forma crítica el slutshaming (tildar de prostituta), fat shaming (insultar por sobrepeso) y el cyberacoso, poniendo en relevancia el aspecto coeducativo que impregna todas las tramas. Aborda la presión social sobre la virginidad, el aborto en una menor, la diversidad de estructuras familiares o la muerte de un familiar.

El término "guerra de sexos» se presenta alejado de las convencionalidades del concepto, eliminando la rivalidad hombre-mujer, y dejando el término como una mera noción deportiva, siendo una nueva ruptura sobre roles y estereotipos comunes (Belmonte y Guillamón, 2008) En la serie se muestra como un grupo de adolescentes se unen para enfrentarse a diferentes retos, muchos de ellos discriminatorios, pero también como dos mujeres son capaces de enfrentarse a cualquier puesto directivo, y buscan entre ellas cierto grado de complicidad (sororidad). En cambio, se observa que cuando las mujeres acceden o se les ofrece cargos superiores, se pone en duda tanto su valía, y su forma de llegar. El estudio recoge la evolución de las series, las cuáles son más cercanas a la realidad, visibilizan problemáticas que se presentan las mujeres y reflejan imaginarios cercanos a la realidad o deseosos de ellos. A pesar de 
los avances, hay una limitada representación de papeles más variados, como étnicos o sociales (Gavilán, Martínez-Navarro, y Ayestarán, 2019). La mayor presencia femenina en ámbitos profesionales va a favorecer un punto de vista más real de la perspectiva de género en las producciones de ficción, como es el caso de la serie "Las del Hockey" creada por Ona Anglada, Laura Azemar, Natalia Boadas y Marta Vivet u otras series de éxito como el "Orange is the new black" de Jenji Kohan. Las series, como el cine, se configuran como un instrumento para difundir contenidos, y como tal, obedece a las intencionalidades de los creadores y la industria, conocedores por otra parte de las demandas de los espectadores y de la realidad social (Ramírez-Macías, et.al., 2011)

Esta serie enriqueció su influencia con su proyección transmedia en redes sociales como Twitter e Instagram, fomentando la cultura participativa entre su audiencia y dejando atrás el papel como consumidoras y consumidores pasivos. A través de sus distintos perfiles se ha ido fomentando el debate y diálogo ante las distintas tramas como las relaciones abiertas, el nivel de compromiso, etc., que se desarrollaban en los diferentes episodios y donde se desprendía un discurso que buscaba trabajar la coeducación hablando al público adolescente de las mismas vivencias que están viviendo.

\section{Conclusión}

Medios de comunicación, televisión y series de ficción tienen un papel socializador en una etapa adolescente donde estamos inmersos en la construcción social e identitaria, tanto individualmente como colectivamente. También es importante resaltar el aumento de series de ficción protagonizadas por adolescentes, aumentando la diversidad de nichos y profundizando en temáticas inexploradas o poco abordadas. Hemos podido constatar que las series han sido testigo de las transformaciones llevadas a cabo en la sociedad como es la evolución a una mayor igualdad de género, en los derechos y en el acceso de la mujer al ámbito laboral. Podemos observar una mayor representación de narrativas desde una perspectiva feminista, pero, pesé a ello, en numerosas producciones perduran roles y estereotipos de género basados en la discriminación.

Las series de ficción pueden reforzar actitudes discriminatorias, pero también puede influir en la desaparición de estos, constituyendo una herramienta educativa para luchar contra las desigualdades sociales y de género. Para ello es necesario una coeducación mediática, tratándose temáticas que permitan la reflexión, la visualización de la igualdad de género y el análisis de contenidos desde una actitud crítica. El uso de las redes sociales como continuación de la trama fomenta el debate entre iguales, y permite configurarse un talante dialógico como «emirec».

A través del análisis y las entrevistas realizadas, hemos podido comprobar que «Las del Hockey» es una serie de adolescentes que apuesta por utilizar un tono coeducativo y reivindicativo, visibilizando temas que no son abordados en las producciones juveniles desde una perspectiva de género, como por ejemplo el consentimiento, el abuso sexual en la pareja, la discriminación del deporte femenino, la presión social para las relaciones sexuales. La sororidad se hace presente en la serie en forma de conversación, de lucha por una causa común o de apoyo ante las adversidades. También la "guerra de sexos", tan utilizado en otros formatos, se transforma en una pequeña 
disputa deportiva, queriendo tensar la posición de la sección masculina en la defensa de sus amigas.

En el metraje audiovisual de la serie vemos acciones reivindicativas contra los estereotipos de género y roles. Dentro del universo de la serie podemos ver situaciones más cercanas a la realidad, pero también imaginarios que son deseados en materia de igualdad. Detalles con que pueden incentivar reflexiones sobre la ruptura de estereotipos como la pertenencia de videojuegos de carreras por parte de una protagonista (Flor), la decisión de dejarse depilarse las piernas (Emma), critica al lenguaje no inclusivo al apellidar siempre a la sección femenina y no a la masculina (Olímpico y Olímpico Femenino) o la infidelidad femenina. También nos encontramos tramas comunes con otras series de ficción, como la virginidad, la muerte de un ser querido, el divorcio de los progenitores, ser madre soltera mediante fecundación 'in vitro' o inseminación artificial, adopción o el aborto. La diversidad de papeles femeninos deja de lado las linealidades de la historia de la ficción, quitando el foco en el hombre y mostrando el pequeño universo de las jóvenes desde una perspectiva de género.

La serie supone un medio para educar en igualdad desde el visionario de la serie, la interacción con sus iguales o actividades que permiten una mirada crítica y reflexiva sobre aspectos reflejados en ella. A su vez, es una herramienta que permite tratar la coeducación en espacios educativos formales y no formales, o en el propio ámbito familiar, creando una atmósfera que invita a la reflexión y al diálogo.

Las futuras investigaciones podrían analizar la percepción de la adolescencia y la identificación con las protagonistas de la serie, así como hacer un profundo análisis de la brecha de género del catálogo de Netflix u otras plataformas. En el ámbito educativo, se podría analizar la percepción de los alumnos y alumnas, antes y después de ver la serie.

\section{Bibliografía}

Anglada, O., Azemar, L., y et al. (2016). «Les de l'hoquei», Trabajo Fin de Grado. Barcelona: Universidad Pompeu Fabra. Recuperado el 1 de septiembre de 2020, de http://hdl.handle. net/10230/27457

Bechdel, A. (1986). Dykes to Watch Out For. Ithaca: Firebrand Books.

Belmonte, J. y Guillamón, S. (2008). "Coeducar la mirada contra los estereotipos de género en TV». Comunicar: Revista científica iberoamericana de comunicación y educación. 31, 115-120. https://doi.org/10.3916/c31-2008-01-014

Belmonte, J. (2014). «Del arte cinematográfico a la imagen postelevisiva: coeducación audiovisual ante las representaciones de la feminidad». Dossiers Feministes, 19, 149-167. Recuperado el 7 de septiembre de 2020, de http://www.e-revistes.uji.es/index.php/dossiers/ article/view/1452/1431

Bermejo, J. (2012). «Los personajes y las series de ficción en la vida de los y las jóvenes». Revista de Estudios de Juventud, 96, 31-49. Recuperado el 5 de septiembre de 2020, de http://www. injuve.es/sites/default/files/Revista96_2.pdf

Bonavitta, P. y De Garay, J. (2011). "De estereotipos, violencia y sexismo: la construcción de las mujeres en los medios mexicanos y argentinos". Anagramas Rumbos y Sentidos de la Comunicación 9 (18), 15-30. Recuperado el 1 de septiembre de 2020, de https://revistas. udem.edu.co/index.php/anagramas/article/view/472

Chicharro, M. (2013). "Representaciones de la mujer en la ficción postfeminista: 'Ally McBeal', 'Sex and the City'y 'Desperate Housewives'». Papers, 98 (1), 11-31. https://doi.org/10.5565/ rev/papers/v98n1.469 
Colás Bravo, P., y Villaciervos Moreno, P. (2007). «La interiorización de los estereotipos de género en jóvenes y adolescentes». Revista de Investigación Educativa, 25(1), 35-38. Recuperado el 9 de septiembre de 2020, de https://revistas.um.es/rie/article/view/96421

Coller, X. (2005). «Estudio de casos». Cuadernos metodológicos, 30. Madrid: Centro de Investigaciones Sociológicas.

Corbetta, P. (2003). «Metodología y técnicas de Investigación Social». Madrid. McGraw-Hill.

Franco, M. B. (2019). «Entrevista a Marina Subirats». Forum Aragón: revista digital de FEAE-Aragón sobre organización y gestión educativa, 26, 39-43. Aragón: Forum Europe de Administraciones de Educación-Aragón.

Galán-Fajardo, E. (2007). «Construcción de género y ficción televisiva en España». Comunicar, 15(28) 229-236. https://doi.org/10.3916/C28-2007-24

Galeón, M. (2019). "La sexualización de la mujer en las series de televisión españolas: Los Serrano, Física O Química y Élite». Trabajo Fin de Grado. Valladolid. Universidad de Valladolid. Recuperado el 5 de septiembre de 2020, de http://uvadoc.uva.es/handle/10324/39689

García, A. y Calleja, B. (2006). Educándonos frente a la televisión. Herramientas para la reflexión y análisis de los contenidos sexistas. Confederación Española de Padres y Madres de Alumnos (CEAPA).

García-Muñoz, N. y Fedele, M. (2011a). «Las series televisivas juveniles: tramas y conflictos en una "teen seriem, Comunicar, 37(21), 133-140. Recuperado el 30 de agosto de 2020, de https://www.revistacomunicar.com/pdf/comunicar37.pdf

Gavilán, D., Martínez-Navarro, G., y Ayestarán, R. (2019). «Las mujeres en las series de ficción: el punto de vista de las mujeres». Revista Investigaciones feministas 10(2). 367-384. https:// doi.org/10.5209/infe.66499

Gerbner, G., Gross, L., Morgan, M., y Signorielli, N. (1986). «Living with television: The dynamics of the cultivation process". Perspectives on media effects, 17-40. Hilldale, NJ: Lawrence Erlbaum Associates.

Gil-Quintana, J. y Osuna-Acedo, S. (2020). «Transmedia Practices and Collaborative Strategies in Informal Learning of Adolescents». Social Sciences, 9(6), 92. https://doi.org/10.3390/ socsci9060092

Gil-Quintana, J., Parejo, J.L., y Cantillo-Valero, C. (2020). Investigar en comunicación y educación. Teoría y práctica científica. Valencia: Tirant Lo Blanch.

Hernández-Carrillo, C. (2016). "Análisis de la violencia de género en adolescentes en la ficción televisiva actual: El caso de 'Por trece razones'. Libro de Actas del II Congreso de jóvenes investigadorxs con perspectiva de género (Getafe, 26 y 27 de junio de 2017). Instituto de Estudios de Género. Madrid: Universidad Carlos III de Madrid.

Igartua, J. J. y Vega, J. (2014). "Ficción televisiva, edu-entretenimiento y comunicación para la salud. Revista de Estudios de Juventud, 106, 15-29. Recuperado el 4 de septiembre de 2020, de http://www.injuve.es/sites/default/files/2014/47/publicaciones/1Ficcióntelevisivayeduentretenimiento.pdf

Mancho-Iglesia, A. y Marta-Lazo, C. (2020). «Audiovisual narratives about the case Spain`s stolen babies». Discourse \& Communication, 12. https://doi.org/10.1177/1750481319893755

Navío Navarro, M. (2018). «El feminismo en las series de ficción: perspectivas sobre el rol de la mujer en The Sinner y Alias Grace». Sánchez-Gutiérrez (Ed.), Feminismo, investigación y comunicación: una aproximación plural a la representación de las mujeres (pp. 13-36). Sevilla: Egregius.

Osuna-Acedo, S., Gil-Quintana, J., y Cantillo-Valero, C. (2018). "La construcción de la identidad infantil en el Mundo Disney». Revista Latina de Comunicación Social, 73, 1284-1307. https://doi.org/10.4185/RLCS-2018-1307|

Perelló, M.A. (2019). "La influencia de las series en la identidad femenina». Trabajo Fin de Grado. Universitat de les Illes Balears. Recuperado el 7 de septiembre de 2020, de http:// hdl.handle.net/11201/152280

Peréz, F. (2019). "Sex Education: La ESI en Netflix». Revista Comunicación y género, 2(1) 2019, 121-134 
Ramírez-Macías, G., Piedra, J., Ries, F., y Rodríguez, A. (2011). «Estereotipos y roles sociales de la mujer en el cine de género deportivo". Teoría de la Educación. Educación y Cultura en la Sociedad de la Información, 12, 82-104. http://dx.doi.org/10.14201/eks.8274

Ruíz, M.J., y Pérez-Rufí, J.P. (2020). "Hermanas, amigas y compañeras en serie. La ficción coral femenina española de las televisiones generalistas y plataformas VOD (1990-2019)». Estudios sobre el Mensaje Periodístico 26(2), 807-826. https://doi.org/10.5209/esmp.67828

Raya, I., Sánchez-Labella, I., y Durán, V. (2018). «La construcción de los perfiles adolescentes en las series de Netflix Por trece razones y Atípico». Comunicación y Medios, 37. http://dx.doi. org/10.5354/0719-1529.2018.48631

Stake, R.E. (2010). Investigación con estudio de casos. Madrid: Ediciones Morata.

Tufte, Thomas. (2004). «Eduentretenimiento en la comunicación para el vih/sida más allá del mercadeo, hacia el empoderamiento». Investigación y Desarrollo. 12. Recuperado el 29 de agosto de 2020, de https://www.redalyc.org/articulo.oa?id=26800102

Verdú, A. D., y Briones, É. (2016). «Desigualdad simbólica y comunicación: el sexismo como elemento integrado en la cultura». La ventana. Revista de estudios de género, 5(44), 24-50. Recuperado el 20 de agosto de 2020, de http://www.scielo.org.mx/scielo.php?script=sci_ arttext\&pid=\$1405-94362016000200024\&lng=es\&tlng=e 